\title{
SYMPTOMATIC EPIPHORA*
}

\author{
BY \\ H. BASIL JACOBS \\ London
}

IN the wide field of possible causes of watering of the eye, if the obvious reflex causes be excepted, the most usual diagnosis is that of a blocked nasolacrimal duct, but since many patients do not fall into this category, an investigation of these problematic cases has been carried out and the findings are described below.

\section{Definitions}

The term "blocked naso-lacrimal duct" is applied so freely that its worth as a diagnostic label has been lost. One criterion is the failure of a dye to drain from the conjunctival sac to the nose, but in Great Britain a more usual criterion is failure or difficulty in syringing the naso-lacrimal duct. Duke-Elder (1952) suggests that a need for force in syringing the tear passages indicates the presence of a "stricture", but there is no general agreement on this point, because various grades of patency may occur and there is more to consider than whether the duct is "blocked" or "not blocked". In the present work such blockages have been divided into complete and partial.

The clinical use of the term "stricture of the canaliculus" is also ill-defined. Many surgeons use it to mean that it is not possible to pass a fine probe through the canaliculus into the sac; to others the term may imply failure to syringe a fluid into the sac by way of the canaliculus and failure of a dye to drain from the conjunctiva to the lacrimal sac. In this paper the failure of a fluid to pass through the canaliculus on syringing is used as the criterion for a stricture of the canaliculus.

\section{Pathology}

There is no satisfactory evidence concerning the pathology of the blocked duct and no histological demonstration of a stricture has been described in the literature. In the absence of histological examination, one may presume a simple congestion of the mucous membrane followed by chronic inflammation at the narrow points of the tearway (Valière-Vialeix, 1939), which may lead to fibrosis. Sondermann (1923) assessed the incidence of stenosis by probing in 55 cadavers (50 men, 4 women, and one child aged 2 years), but it is not recorded that any of these subjects suffered from epiphora in life. Sondermann found that 6 to 7 per cent. of canaliculi showed marked and 13.3 per cent. slight stenosis; 40 per cent. of naso-lacrimal ducts showed considerable, 29 per cent. moderate, and 31 per cent. no stenosis. These stenoses were regarded as pathological, but it is hard to understand this high frequency in apparently normal, mostly male subjects, and it seems safer to conclude that the patency of the canaliculi and naso-lacrimal duct as evidenced by probing varies widely. In the cadavers of four patients who actually had dacryocystitis 
the bony naso-lacrimal canals were found to be more narrow and winding than in normal subjects

Seidenari (1947), discussing the submucosal venous network and its resemblance to erectile tissue, suggested that its turgescence might lead to an adhesion which becomes permanent. Duke-Elder (1952), commenting on the positive family history found in many patients with watery eyes, suggests that the inherited factor (to which such epiphora is secondary) may be some anatomical peculiarity of the soft tissues or bone. Seidenari also reported the microscopical findings in sacs removed in the course of eight dacryocystectomies; six were quite normal and two showed definite inflammatory changes.

Our scanty knowledge is due to the difficulty of obtaining pathological material from affected cases, the lacrimal sacs that are removed being above the usual site of a block.

\section{Methods of Investigation}

Since some of the diagnostic procedures available are also therapeutic measures, the usual practice of investigating the patient as far as possible at his first visit may make it difficult to decide which particular line of treatment has been beneficial, and the final diagnosis may remain in doubt despite a clinical cure.

A routine of questioning and examination on the lines indicated below has been used as the basis of the present inquiry:

(1) History.-This should include the age and sex of the patient, mode of onset, precipitating causes, family history, occupation and hobbies, history of nasal or other disease, menopause, and the presence of any irritative symptoms.

(2) Examination.-A careful general examination should include the appearance and configuration of the face and nose, the complexion, the presence of dilated facial veins, and evidence of skin disease of the face or scalp.

Examination of the eyes should include the position of the globe (whether enophthalmic or exophthalmic), the position of the lids (whether turning in or out, whether the lower lid is retracted or not), the tone and strength of the orbicularis oculi (by digital estimation), and particularly the position of the lower punctum, a small displacement of which is a common cause of epiphora in elderly people. The cleanliness or otherwise of the lashes may give a clue to the patient's standard of hygiene and to possible external sources of irritation such as papillomata or molluscum lesions of the lash line. The presence of redness, inspissated discharge, or squames at the roots of the lashes, and the direction of growth of the lashes should also be observed.

The normal appearance of the conjunctiva shows a confusing variation, but an attempt should be made to assess any significant redness and a comparison should be made with the contralateral conjunctiva and with the patient's facies. The caruncle frequently seems to be a sensitive indicator of irritation, but it is often more hyperaemic in the symptomless patient than the rest of the conjunctiva and thus again assessment of its normality is extremely difficult. The presence of foreign bodies or concretions will be evident.

A further revealing point may be a very slight degree of conjunctival oedema, not necessarily associated with redness, which is suggestive of an allergic factor.

A foreign body or a faint punctate staining with fluorescein are possible corneal affections for which a search must be made.

(3) Bacteriology.-Before any drops are instilled into the eye a smear and a culture should be taken to determine the nature of the cellular exudate and the presence of in- 
fection. Should any organism be grown, its sensitivity to the available antibiotics can be tested.

(4) Dye Test.-This involves observing the rate of disappearance of a drop of fluorescein ( 2 per cent. in water) instilled into the conjunctival sac. In normal subjects, there is a marked decrease in the yellowness of the conjunctival fluid so stained, within 1 to 2 minutes. This indicates that the tears have drained, and a yellow staining of the nasal mucus on blowing the nose or a staining of the material coughed up from the back of the throat will confirm that the tears have passed down the naso-lacrimal duct. The two eyes may be tested separately or simultaneously and the rate of disappearance can be compared.

The rate of drainage may be normal and speedy, slow, or doubtful, or there may be no drainage at all.

If the dye overflows the lid margins, the lids should be cleaned and the test repeated to avoid a misinterpretation of the result. Not infrequently the dye will disappear at a nearly normal rate and yet the naso-lacrimal duct may be quite blocked on attempted syringing. This false positive result occurs when there is a voluminous mucocoele into which the fluorescein can drain freely; this may be confirmed by pressing over the sac when a yellowstained discharge will be regurgitated.

The dye test is usually used to verify the patency and function of the lacrimal passages as a whole, but if the naso-lacrimal duct is blocked, it can be used to assess the function of the canaliculus. In the presence of a mucocoele, this is easily done as indicated above by expressing the sac after the dye has been instilled into the conjunctiva. If no mucocoele is present, the conjunctival sac should be irrigated to remove any excess dye a minute or two after the dye has been instilled. The operator then syringes through the lower canaliculus with saline, whilst an assistant everts the upper punctum and holds a pledget of cotton wool just away from it to catch the first stream of regurgitating fluid. A yellow staining of this fluid will indicate that some dye is likely to have drained into the sac and that the canaliculus is functioning normally. It is possible that the coloration could be derived from the dye lingering in the canaliculus itself but the test maybe regarded as a valuable pointer.

(5) Schirmer's Test.-This is described by its originator as follows:

"I use blotting-paper strips $0.5 \mathrm{~cm}$. wide and $3.5 \mathrm{~cm}$. long. At one end a $0.5 \mathrm{~cm}$. piece is bent over, and the two corners are cut off so that it does not stick into the conjunctiva. The conjunctival sac is swabbed out in the event of its being abnormally full, and the bent piece is carefully pushed behind the slightly drawn down lower lid upon both eyes*. The two strips remain here for 5 minutes, and then the length of the section moistened during that time is noted. Should the entire strip be wet through before the expiration of 5 minutes, the time required for this is noted.

"Investigations upon considerably more than one hundred normal eyes have shown me that, on the average, 5 minutes is just sufficient to moisten the whole strip. However, it also happens that the strip is moistened in one, even in half a minute, while, on the other hand, the secretion may sink to 6 to $7 \mathrm{~mm}$. in 5 minutes-the piece lying in the conjunctival sac is never included in the measurement; such cases, however, are rare, and a paresis of the excito-lacrimal fibres is to be suspected in every patient in whom the moistening of the strip amounts to less than $1.5 \mathrm{~cm}$. in 5 minutes. A moistening of more than $1.5 \mathrm{~cm}$. guarantees, on the contrary, normal glands, and in such patients the examination is herewith ended.

"The others must now be examined by a second method. The patient receives in each eye three drops of a 4 per cent. solution of cocaine at intervals of one minute-in order to eliminate the stimulus which in Method 1 is effective only from the conjunctiva; then the strip as formerly is inserted on the right, and a camel hair brush is pushed high up into the right nostril, where rotatory movements are carried out with it. The lacrimal gland, excited reflexly thereby, now secretes so freely that in two minutes on an average, the entire strip becomes wet. In general, this method yields more constant results in the same hands than the first, presumably because the 
excitability of the nasal mucous membrane does not vary within such wide limits as does that of the conjunctiva; fluctuations, however, naturally also occur here. The secretion may be very much more vigorous, so that within half a minute the whole strip is moistened, or it may proceed very much more slowly. However, I have never found a normal eye in which the length of moistened strip amounted to less than $1.5 \mathrm{~cm}$. in 2 minutes, so that eyes with still less secretion may be regarded as diseased.

"With this method are found reliable data and a numerical expression for the diminished functional power of the gland." (Quoted from Sjögren, 1943).

The second part of the test was not used in the present investigations. The only modification was the insertion of the strip just lateral to the punctum, which was held slightly everted to avoid drainage of the tears during the test.

In a series of normal adults a mean value of $14.5 \mathrm{~mm}$. of watering in women and 13.1 $\mathrm{mm}$. of watering in men with a wide scatter from nil to over $30 \mathrm{~mm}$. has been found (Fig. 1, opposite).

The secretion tended to be less in older patients. In 23 per cent. of men (16 of 69) and 22 per cent. of women $(23$ of 103$)$ there was a difference of secretion of $6 \mathrm{~mm}$. or more between the two eyes. The average of the differences in these cases was just over $9 \mathrm{~mm}$. for both sexes (Table I).

TABLE I

RESULTS OF SCHIRMER'S TEST

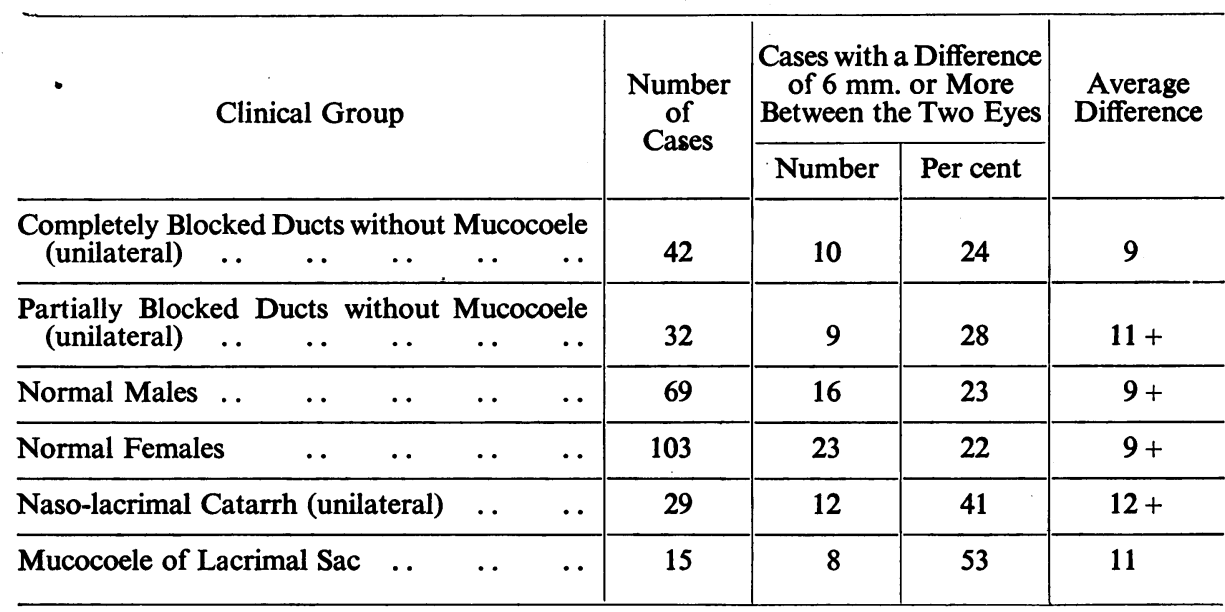

Controls of all ages were used, but it was found that some eyes were so irritable that the paper was completely wetted in a few seconds and these subjects, mostly young people, were excluded. The figures given above are somewhat lower than those established by previous workers, and the exclusion of these eyes may explain the discrepancy.

The assessment of hypersecretion by this rough test is not reliable, but a difference between the results obtained in the two eyes is more significant than an indication of hypersecretion in both. It is regrettable that this test seldom serves to distinguish between eyes which water because of excess tear secretion and those which water because of defective drainage.

(6) Orbicularis Oculi.-Dynamometric estimation of the strength of the orbicularis oculi was done by the simple method already described (Jacobs, 1954). The average finding is equivalent to $120-140 \mathrm{~mm}$. $\mathrm{Hg}$ with a wide variation above and below these figures. Similar findings were obtained in a group of normal students. 


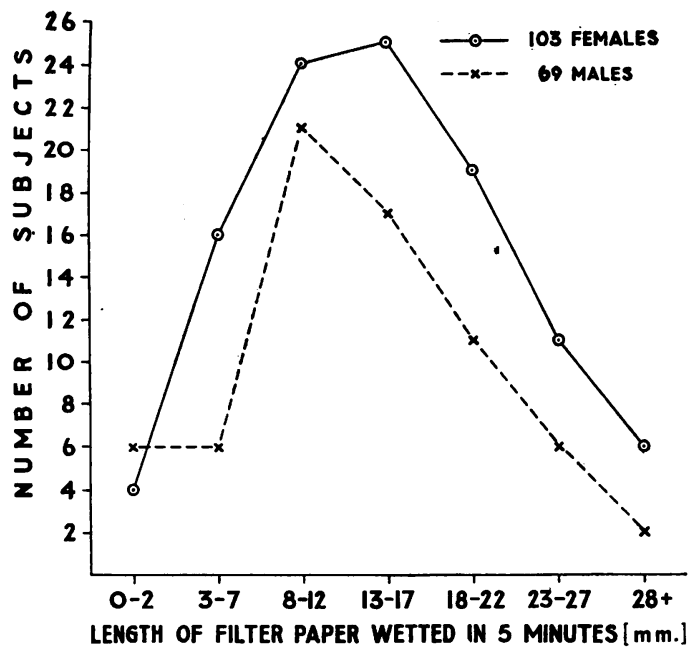

FIG. 1.-Result of Schirmer's test in normal subjects.

Although the assessment of the incidence of weak muscles in this way may reflect poor muscle tone, it is not as significant as a direct measurement would be. It is unfortunate that there is no recognised way of doing this.

(7) Faradism.-The response of the orbicularis oculi to faradism may also be determined with the object of assessing any weakness of that muscle. Sometimes the response may be diminished in vigour for a standard stimulus and a comparison of the normal with the abnormal may be valuable. A source of error in this test is the variable skin resistance that may be encountered and the results obtained must be viewed critically. It has not been found very helpful.

(8) Syringing.-The lower canaliculus was syringed with saline with a 2-ml. Record Syringe after the punctum had been dilated with a conical dilator. The saline may flow freely to the back of the throat, may regurgitate through the upper punctum or around the cannula, or may not flow at all. If the flow is not free, the cannula should be advanced into the sac to ensure that the whole canaliculus is open. If the flow is free, then the anatomical integrity of the whole lacrimal pathway from the punctum to the inferior orifice in the nose is confirmed. If part of the fluid flows to the back of the throat and some regurgitates, it is still justifiable to draw the same favourable conclusion.

The function of the canaliculus is tested with fluorescein by syringing as described in Section (4) Dye Test. If drainage is poor and the canaliculus is thus demonstrated to be working normally, the cause can only lie in some defect in the naso-lacrimal duct, or at its entrance into the nose. This test has been found inconsistent at times, but is nevertheless valuable, especially if positive.

Should the fluid regurgitate from the upper punctum, then a block may be sited in one of two positions.

If a block is present as in Fig. $2 a$ (overleaf), a coincident block as in (b) can be excluded by hypodermic injection of the sac with saline or Novocain, thus by-passing the canaliculi altogether.

Should the regurgitation take place round the cannula, the block may be sited as in Fig. $2(c)$. 

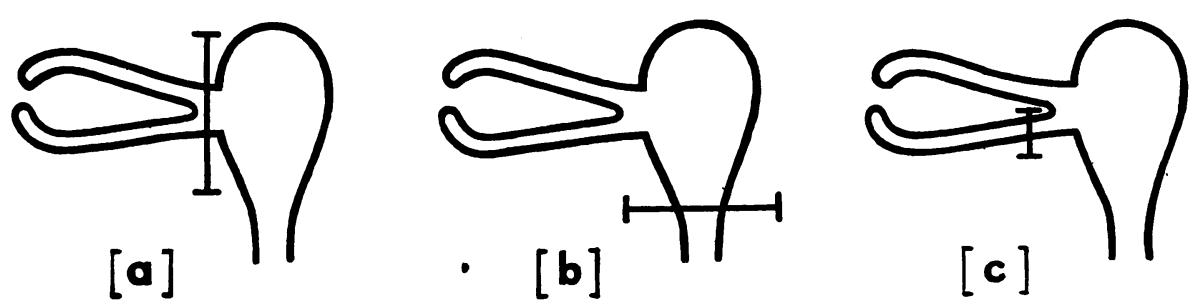

FIG. 2.-Possible positions of blockages.

Syringing through the upper canaliculus will be quite easy in this third instance, unless there is also a block there as well. In some cases, blocks are found at both $(b)$ and $(c)$; on the other hand, a block in the canaliculus may be excluded if the sac is felt to dilate as pressure is exerted on the piston of the syringe. Failure of even the finest probe to enter the sac is not enough to justify the diagnosis of a stricture in the canaliculus, as a normally functioning canaliculus often will not allow the passage of an instrument through it. The above tests coupled with dacryocystography are necessary to establish this diagnosis.

(9) Pressure Syringing.-This is a modification of the method used above when regurgitation through the upper punctum occurs. In this event, the reflux can be prevented by blocking the upper punctum with a conical dilator. The fluid may flow to the back of the throat, freely, with difficulty, or not at all. If regurgitation takes place around the cannula, a conical model on the Anel pattern may be used.

The test of pressure or forced syringing should be repeated at least once before the diagnosis of a completely blocked duct is made.

Many naso-lacrimal ducts that are labelled blocked by ordinary syringing will be found to be patent by the use of pressure and by preventing regurgitation with a conical dilator. Four grades of patency may be distinguished:

A. Freely patent to simple syringing.

B. Freely patent to pressure syringing

C. Patent with difficulty to pressure syringing $\}$ partially blocked duct.

D. Not patent at all to any method of syringing-completely blocked duct.

Pressure syringing is no more dangerous than simple syringing (Wollenberg, 1927); if pain indicates that the sac is becoming overdistended the procedure should be abandoned. Two infrequent complications are a leak into the tissues and a valvular overdistension of the sac; but both can and do result from simple syringing. A leak into the tissues is unimportant unless sepsis occurs, and this can be avoided by the regular instillation of Albucid drops into the eye for a few days. In many hundreds of pressure syringings, sepsis from a leak of saline into the tissues has never occurred. More distressing is the condition in which the sac is overdistended so that it presses on the canaliculus and prevents emptying. Two cases of this condition which occurred after the simple syringing of a mucocoele settled down after the local applications of an ice pack to the sac and vasoconstrictors to the nose. However, it is very painful and unpleasant, and should be avoidable if a finger is pressed on the sac whilst it is being syringed.

(10) Measurement of Intercanthal Distance.-The distance between the muco-cutaneous junctions of the two medial canthi was measured with the Bishop Harman rule. The normal range was found to vary from $25-40 \mathrm{~mm}$.; the usual reading was between 28 and 36 $\mathrm{mm}$., the mean in men being $31.6 \mathrm{~mm}$. and in women, $30.8 \mathrm{~mm}$. 


\section{Clinical Investigation}

The methods described in the previous section were used in the investigation of 350 adult patients who suffered with watering of the eyes. The proforma shown in Table II was used for each patient. Although the object of the work was to distinguish the aetiology of epiphora in the absence of an obvious reflex cause or a blockage of the duct, certain patients thought to have a blocked duct were included for the sake of comparison.

TABLE ॥

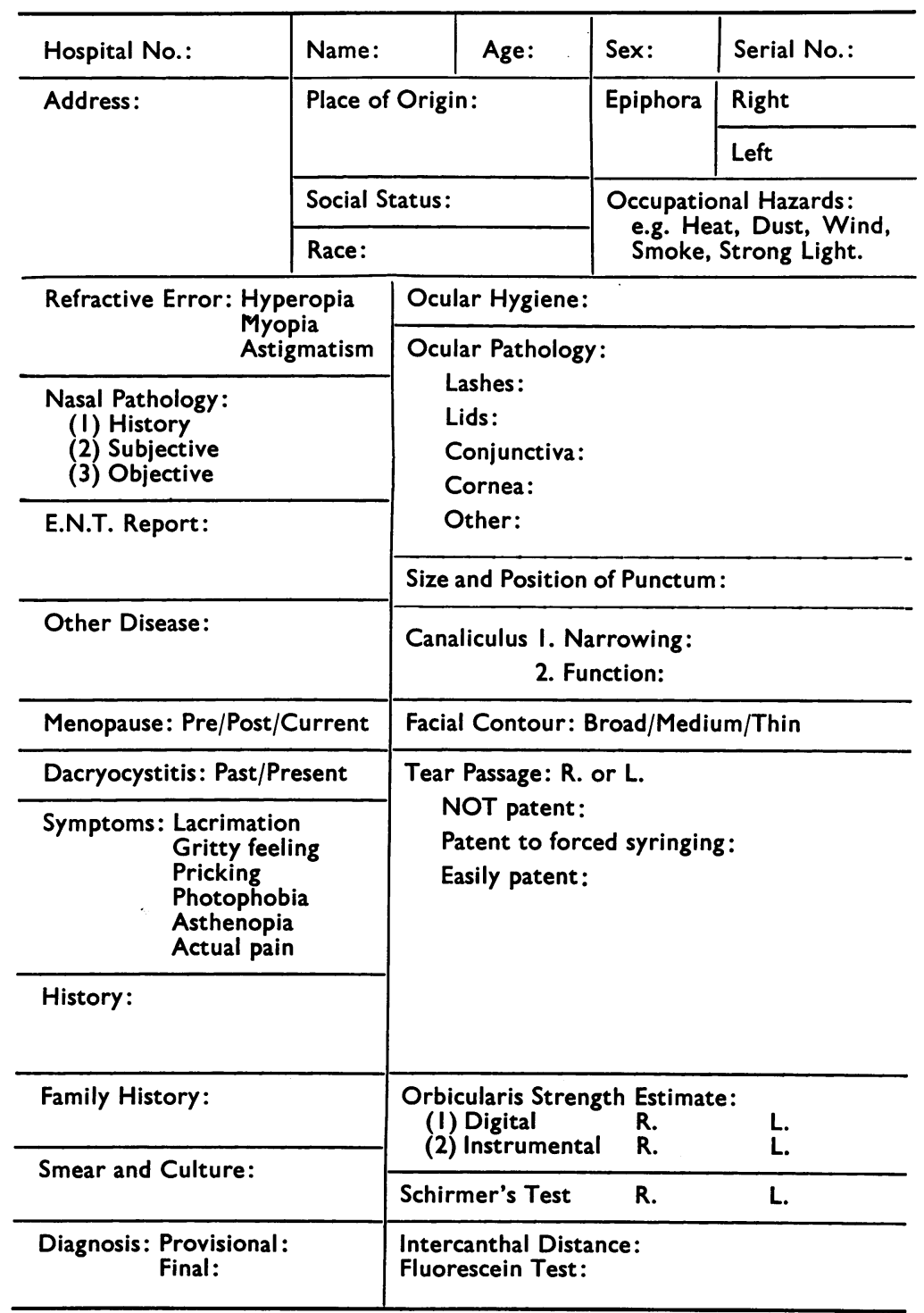


As the investigation proceeded, many patients in whom it had been difficult to make a diagnosis were thought to be suffering from "naso-lacrimal catarrh", and the following classification was made:

(1) Blocked naso-lacrimal duct (complete or partial)

(2) Naso-lacrimal catarrh

(3) Undiagnosed (N.Y.D.)

Apart from these major groups, there were smaller sub-groups and even individual cases sufficiently interesting to deserve separate comment.

(1) Blocked Naso-Lacrimal Duct.-In this series there were 105 cases, 58 completely blocked and 47 partially blocked.

General Considerations.-Females outnumbered males in this series by roughly $3: 1$, the proportion in completely blocked ducts being $4+: 1$ and in partially blocked ducts $2: 1$. The sex ratios reported in the literature vary from three or four females to one male. The slightly atypical findings with Schirmer's test for the group with partial blockage are indicated in Table I and suggest a heterogeneous factor in this group.

The age group 40-55 years is chiefly affected; the detailed incidence for females is shown in Fig. 3 (opposite), but the males were too few for the numbers to be plotted. The men showed a similar type of incidence to the women, but there were more cases in the 66-70 age group than would be expected. Fig. 3 shows that patients with partial block and complete block attended the clinic in equal numbers up to the age of 54, but that there was a sudden drop in the numbers of partially blocked ducts in patients of 55 and over.

Throughout this paper, it will not be possible to comment on the racial and social pattern of the patients, as all were seen in hospital and the racial variations probably reflect those of the district served by the hospital. The literature shows differences of opinion concerning the social standing of the patients, but it is agreed that Negroes are relatively spared.

The right eye was affected 53 times, and the left eye 37 times in ninety unilateral cases; only fourteen cases were bilateral.

The length of history varied from 2 months to 30 years for complete blocks and from 4 months to 20 years for partial blocks. The range is so wide that no purpose is served by analysing the figures.

Positive family histories were forthcoming in seventeen of 57 patients with complete blocks and in six of 47 patients with partial blocks (30 and nearly 13 per cent. respectively).

The incidence of local irritative symptoms was the same in men as in women: thirteen of 57 cases of complete block and thirteen of 47 cases of partial block. Occupational hazards, the significance of which is difficult to assess, were more common in men than in women, as one would expect.

Questions concerning the general health revealed that the incidence of an artificial menopause, gastric troubles, and skin disorders did not seem to be particularly significant.

A history of nasal disorder was given by 21 of 47 patients with a partial block and by 23 of 58 patients with a complete block, in comparison with only 53 of 208 


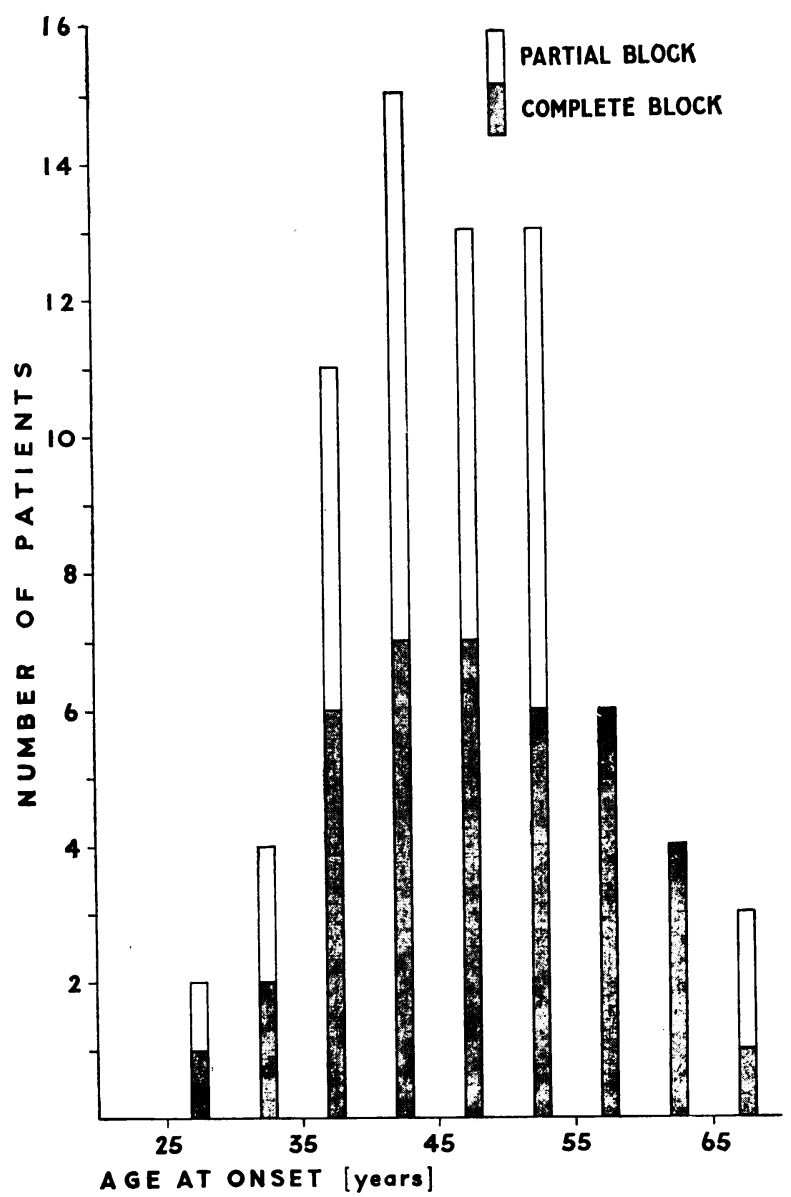

FIG. 3.-Age incidence of blocked naso-lacrimal duct in females.

controls. It is of interest that 23 of 123 medical students gave a similar positive history of nasal disorder.

A precipitating factor was reported by ten of 58 patients with a complete block; seven occurred after some ocular inflammation or irritation, two after nasal infection, one after a Bell's palsy with complete recovery, and one after a cyst of the lid. Only four of 47 patients with a partial block reported a precipitating factor-one Bell's palsy with complete recovery, one attack of dermatitis of the face, one corneal ulcer, and one new pair of spectacles.

Bacteriology.-In a limited series of bacteriological studies, pathogenic organisms and pus cells were seen six times and non-pathogens, no growth, and/or no pus cells 27 times.

Strength of the Orbicularis Oculi.-This was found to be within normal limits in the group of cases with partial blockage. In patients with a complete blockage the mean reading was lower than would be expected, perhaps because of the 
increased need to dry the eyes and consequent weakening of the muscle by a wiping action.

Intercanthal Distance.-In all types of blocked duct, regardless of sex, these measurements were similar to those found in normal subjects. The inference that complete blockage is not necessarily related to narrow bony passages is confirmed by recent radiographic investigations (Phillips and George, 1956).

Schirmer's Test.-In cases of complete blockage the mean values given by this test $(16.6 \mathrm{~mm}$.) were higher than in the normal group. In ten of 42 unilateral cases there was a difference of more than $6 \mathrm{~mm}$. on the affected as compared with the unaffected side (the average $9 \mathrm{~mm}$.). There was a similar difference in nine of 32 cases of partial block (average $11+\mathrm{mm}$.) (Table I).

These figures indicate that, in general, there is a significant degree of hypersecretion in cases of blocked naso-lacrimal duct without mucocoele as compared with normal subjects, but if one compares the affected side with the unaffected in unilateral cases (as in Table I), the reality of any appreciable difference in secretion in these cases becomes more dubious. However, eight of fifteen patients (53 per cent.) with a mucocoele and completely blocked duct had an increased tear secretion on the affected side, the average difference being $11 \mathrm{~mm}$.

Thus, some patients with a mucocoele show a significant hypersecretion of tears due to irritation in addition to a defect of drainage. It can be conjectured that the removal of the sac may be attended by a greater relief of symptoms in such patients than in others, for although no drainage mechanism is provided by the operation, the lessening of chronic infection and irritation and, therefore, the lessened productions of tears, will be sufficient to provide a clinical improvement.

Treatment.-Patients with a complete blockage were advised to have a dacryocystorhinostomy and were sent back to the surgeons under whose care they had originally been.

Thirteen partially blocked ducts were subjected to a course of simple syringing: the criterion for the diagnosis in these cases was their good response to syringing, and thus no comment on this treatment is required. The only point of interest is that in six of the seven cases in which a dye test was performed the dye drained away, and that in one the result remained equivocal.

Of 25 patients whose ducts were subjected to a course of pressure syringings, eleven were unimproved and fourteen were cured. Seven of these successes were confirmed by a dye test, three not confirmed, and in the other four, either no dye test was performed or its interpretation was uncertain.

Four partially blocked ducts were probed and this was followed up with a course of syringings. Two of these patients were cured and the result was confirmed by the dye test, and two remained unchanged. One of the latter claimed to be better, although the dye test continued to indicate that no drainage was taking place.

One patient reponded to ephedrine drops.

Thus, it may be concluded that more than half of the patients with a partially blocked duct patent to pressure syringing only will respond to conservative treatment. It is pertinent to point out that these are usually classified as cases of completely blocked naso-lacrimal ducts. 
Not sufficient cases in this group were treated by probing to enable an assessment to be made of the value of this procedure.

(2) Naso-Lacrimal Catarrh.-Many patients who attended the clinic were found to have no obvious blockage of the lacrimal pathways but showed one or a combination of the following characteristics:

(a) a mild conjunctival infection or irritability not obviously outside normal limits,

(b) a suggestive history of conjunctivitis and/or bacteriologically confirmed infection of the conjunctiva,

(c) a satisfactory response to therapy designed either to cure any infection and/or relieve any irritation,

(d) a primary nasal disorder.

In many ways the term "irritable eye" would have been preferable to "naso-lacrimal catarrh", for in most cases the eye appeared to be unduly sensitive. However, as one can logically view the conjunctiva as part of the lacrimal pathway, the term "naso-lacrimal" is a convenient anatomical omnibus to include conjunctiva, canaliculi, lacrimal sac, and naso-lacrimal duct, and a mild congestive or inflammatory affection of one or more of these parts may be termed "catarrh". The clinical picture will vary with the part affected. If only the conjunctiva is affected, the appearance will be that of a low-grade chronic conjunctivitis or irritable eye; if the naso-lacrimal duct is affected, irritability of the eye will be less evident and the cause of the watering more obscure. The canaliculus may accommodate a probe on one day but not on another, and the naso-lacrimal duct may be syringed easily one day and not another; these structures are subject to fairly rapid changes in patency that can be best explained by the presence of congestion in the lining walls.

Ten patients were seen in whom the epiphora seemed to be secondary to nasal disease. A distinction between nasal disease causing epiphora and nasal disease accompanying epiphora can only depend on the opinion of the observer and it is striking that otorhinological opinions on the relation of a condition of the nasal mucous membrane to epiphora seem to depend as much on the observer as on the observed. The nasal mucosa should be examined not only for evidence of serious pathology, but for minor degrees of swelling and congestion that might be relevant to epiphora though hardly worthy of note in their own right. In such a small number the diagnoses can hardly be significant, but they included maxillary sinusitis, and mucous, vasomotor, and hypertrophic rhinitis. An immediate exacerbating feature in two cases was a florid conjunctivitis.

General Considerations. - The sex ratio was nearly equal (52 men and 55 women).

The greatest incidence among the women was in the 45-59 age group, about 5 years later than the average age at onset of blocked naso-lacrimal duct. The men showed one peak of incidence at 40 to 54 years and a second at 65 and over (Table III, overleaf). 
TABLE III

AGE AT ONSET OF NASO-LACRIMAL CATARRH

\begin{tabular}{|c|c|c|c|c|c|c|c|c|c|c|}
\hline Age Group (yrs) & $\begin{array}{c}\text { Under } \\
25\end{array}$ & $25-29$ & $30-34$ & $35-39$ & $40-44$ & $45-49$ & $50-54$ & $55-59$ & $60-64$ & $\begin{array}{l}65 \text { and } \\
\text { Over }\end{array}$ \\
\hline Females .. & 2 & 0 & 1 & 4 & 4 & 9 & 11 & 12 & 6 & 6 \\
\hline Males & 4 & 3 & 2 & 2 & 9 & 7 & 7 & 3 & 3 & 12 \\
\hline
\end{tabular}

There was a preponderance of bilateral cases $(66: 38)$, the proportion in women being $26: 25$ and in men $40: 13$.

The length of history showed a wide range similar to that displayed by patients with a blocked naso-lacrimal duct.

Six men (11 per cent.) and fourteen women $(26+$ per cent.) gave a positive family history.

The incidence of irritative symptoms was similar in men and women and totalled 48 of the 107 cases, being significantly more than in the cases of blocked nasolacrimal duct. Occupational hazards were more frequently noted in men than women.

Ten of the 53 women had had either a hysterectomy or an artificial menopause. Stomach complaints occurred in seven male patients. There were twelve patients with skin disorders-generally rosacea, seborrhoea or psoriasis. A history of nasal disorder was given by 28 men and thirty women, a slightly higher incidence than in the group with blocked ducts.

Many cases were unilateral, or if both sides were affected, one was worse than the other. The right eye was worse in 41 and the left in 31 of about seventy patients whose symptoms could be assessed in this way.

Precipitating factors were cited by fourteen patients (the same incidence as in the cases of blocked duct): nine had had a definite conjunctivitis or red, sore, and discharging eyes; two had had contact with a case of conjunctivitis; one child had had measles; and two had had a stuffy or running nose.

It was thought that a constitutional predisposition in the form of a skin disorder, an unusually rosy complexion, or dilated facial veins might play a part in the causation of symptoms, and eighteen men and twelve women seemed to exemplify this possibility; 21 of these thirty cases were bilateral, and of the nine unilateral cases, six were females. In two or three of these patients, a significant exogenous factor was also present, and one man with seborrhoea capitis also suffered from a manifest anxiety state.

Environmental circumstances gave a clue to a possible exogenous irritable factor associated with an occupation or hobby in twenty patients, most of them men.

Thirty of the 107 patients volunteered information on some exacerbating factor, generally cold air, wind, or a change of temperature, but in six cases emotional strain.

Bacteriology.-Pathogenic organisms were found on culture or pus cells in a smear in eighteen out of 56 tests.

Of seventeen patients (6 men and 11 women) thought to have a low-grade infection of the conjunctiva, bacteriological confirmation was obtained in fifteen; 
two elderly men, one who had had trachoma and one with a history of sticky eyes, yielded no bacteriological evidence of infection. Ten of the seventeen cases were bilateral ( 5 men and 5 women) and seven were unilateral (1 man and 6 women). Four patients were found to have some rhinitis, two had conjunctivitis or had been in contact with a case, one was subject to frequent head-colds, and two were constitutionally predisposed. A high average value of $18 \mathrm{~mm}$. for Schirmer's test was found in this sub-group.

Strength of Orbicularis Oculi.-The average measurements were 100-140 mm. $\mathrm{Hg}$-not conspicuously different from normal.

Intercanthal Distance.-This showed no significant variation from normal.

Schirmer's Test.-The mean value was little above the accepted normal. In twelve of 29 cases with unilateral naso-lacrimal catarrh, the affected side gave higher results than the unaffected side (Table I). Thus in some of these unilateral cases hypersecretion of tears would appear to occur.

Treatment.-The measures taken should be conservative and should include the topical administration of drugs to the conjunctiva, the treatment of nasal disorder, and sometimes systemic medication.

Administration of an antibiotic topically to eliminate infection is a sound first step; an ointment instilled five or six times daily serves the purpose well. Some patients in whom bacteriological confirmation of infection was lacking improved on such treatment. An astringent lotion or vasoconstrictor drops after 10 to 14 days of such antibacterial measures help to soothe the mildly irritated conjunctiva. In sixteen eyes the dye test indicated restoration of normal drainage after treatment and seven were cured by this regime.

Other cases were cured by ephedrine drops applied to the eye, Albucid drops, a bland lotion and lanoline ointment to soften the skin of the lids, a change of occupation, treatment for seborrhoea capitis and a bland eye lotion, short wave diathermy, orbicularis exercises and massage, the antihistamine "Thephorin" taken by mouth.

One patient had received such an assortment of different therapeutic measures that it is not possible to say which of them helped. In twenty eyes the dye test failed to demonstrate restoration of normal drainage despite treatment: two of the patients concerned defaulted. In at least 36 cases, the fluorescein test demonstrated that a defect of drainage apparently played no part so that either the tear secretion was excessive or the patient was unduly troubled by a normal secretion. The dye test was not always unequivocal but some defect was demonstrated in 36 eyes.

The results of Schirmer's test indicate that in some cases undue trouble was caused by a normal secretion. However, it is also possible that the patient had intermittent paroxysmal obstruction with or without intermittent and paroxysmal hypersecretion, so that no abnormality of drainage or secretion was demonstrable at his first attendance at the clinic.

If the patient has a constitutional predisposition, such as rosacea or seborrhoea, this should be managed on general lines. The plethoric patient with a correspondingly reddish conjunctiva may be helped by silvering the lids. Syringing with saline sometimes makes these patients worse. 
Further helpful local measures are short wave diathermy, zinc ionization, and exercises and massage of the lids, all directed at relieving congestion and aiding the venous return. Exercises and massage also help to control the mild eversion of the punctum that may develop in any patient with epiphora. Thephorin tablets were found useful in two cases and have the advantage of being mildly sedative as well as antihistaminic. Much time and patience on the part of the surgeon and the subject may be needed for the prosecution of these conservative measures to a successful conclusion. Those patients with no defect of drainage were not treated in the clinic, but were referred back to their own surgeons for treatment.

(3) Undiagnosed (N.Y.D.).-This section comprises 44 cases which were difficult to diagnose.

General Considerations.-Women outnumbered men by $3: 1$. The age at onset in eleven men varied between 35 and 75 years; that of the 33 women is indicated in Table IV.

TABLE IV

AGE AT ONSET OF FEMALE UNDIAGNOSED CASES

\begin{tabular}{c|c|c|c|c|c|c|c|c|c|c}
\hline Age Group (yrs) & $\begin{array}{c}\text { Under } \\
25\end{array}$ & $25-29$ & $30-34$ & $35-39$ & $40-44$ & $45-49$ & $50-54$ & $55-59$ & $60-64$ & $\begin{array}{c}65 \text { and } \\
\text { Over }\end{array}$ \\
\hline Number of Cases & 3 & 2 & 2 & 2 & 6 & 8 & 0 & 1 & 4 & 5 \\
\hline
\end{tabular}

21 were bilateral and 23 unilateral, there being no marked variation between the sexes.

The length of history varied to a meaningless degree from 6 weeks to 30 years.

A positive family history was forthcoming in one man (10 per cent.) and seven women (21 per cent.).

Irritative symptoms were present in two men and eleven women. Occupational hazards were admitted by three men and six women-a reversal of the usual relative frequency.

A history or rhinologist's report of nasal disorder was given in three men and fourteen women. In objective reports on nine of these cases, six were found to have an organic defect.

The watering was unilateral or worse in one eye than the other in 35 cases, nineteen in the right eye and sixteen in the left.

Various factors were said to precipitate the symptoms in six cases. A corneal foreign body, sticky eyes, blepharitis (?), a bilateral Caldwell-Luc operation (the epiphora was unilateral), a hemiplegia some time before, and herpes zoster ophthalmicus 6 months before, were all volunteered as relevant information in this context.

Bacteriology.-Too few studies were made to deserve mention.

Strength of Orbicularis Oculi.-Within normal limits.

Intercanthal Distance.-Within normal limits. 
Schirmer's Test.-Some of the values given by Schirmer's test in these patients tended to be higher than average, but a mean value of $15.9 \mathrm{~mm}$. for 41 of them is not much different from the accepted mean. A difference of more than $6 \mathrm{~mm}$. between the affected and normal sides was found in four of the nineteen cases (average $10 \mathrm{~mm}$.).

Treatment.-As a rule, the treatment of these cases was unsatisfactory and even when some success was obtained it was not possible to estimate the efficacy of the measures adopted. Some patients defaulted and no blame can be attached to them as the general nuisance of regular hospital attendance must often outweigh the discomfort of a watery eye. A larger proportion attended regularly as requested and responded or failed to respond to various combinations of treatment, the lines of which did not differ materially from those already described for the cases of naso-lacrimal catarrh. There was no doubt that, in a few cases, the symptoms were due to an excessive physiological response to a normal cause for watering. Three patients stated they were worse in the early morning, when a minor degree of moisture of the eyes occurs in many uncomplaining patients. Some mentioned the dependence of the symptom on temperature and wind, two suggested that close work was an exacerbating factor, and one was troubled only when cycling. Wearing spectacles and bending seemed to make the watering worse in two other patients.

Exercises of the orbicularis oculi helped a few patients, but the clinical data was too indefinite for a diagnosis to be made. It was thought that it rested between "weak muscles" and "naso-lacrimal catarrh".

Dye tests were performed in only nineteen patients and a definite defect of drainage was demonstrated in eight; in three patients fluorescein tests demonstrated a restoration of normal drainage after treatment.

One of these "cured" patients was a woman aged 40 with a very rosy complexion who was thought to have an irritable but white eye, and a demonstrable improvement in tear drainage occurred spontaneously. The second was a woman seen at the age of 49 years, who was apparently cured by syringing the naso-lacrimal duct. Three years later she had a relapse and, although she claimed to be better after a similar course of syringing, the dye test failed to demonstrate drainage until after a course of exercises. The third "success" occurred in a woman aged 75 who had mildly red eyes and who defaulted after a course of syringings so that the immediate result was unascertainable. A year later, she was treated for conjunctivitis in another clinic, and $2 \frac{1}{2}$ years later, although she still complained of watering, fluorescein drained away in both eyes, albeit a little more slowly in the right than in the left.

\section{Other Diagnoses}

(a) Muscle Weakness.-Fourteen patients in whom exercises were given to promote the tone and functioning of the orbicularis oculi, have been described in full in a previous paper (Jacobs, 1959).

(b) Disorders of the Canaliculus.-The commonest affection was a stricture, and eight patients so affected were seen. Two of the strictures had occurred 
after irradiation and one after herpes zoster ophthalmicus. The best method of management of these cases is to slit open the occluded canaliculus up to and beyond the stricture. Failing this, the upper canaliculus-provided it be normal-may be slit open along its complete length into the sac. A cantho-dacryocystostomy or cantho-rhinostomy may be attempted if these measures fail. It may be worth while trying to dilate the stricture with a probe, but this measure is rarely efficacious.

One case was seen with Streptothrix infection and another with a pneumococcal canaliculitis. No radiographic studies were attempted in the latter case, which settled down on treatment with an antibiotic, but the patient may have had an infected diverticulum of the canaliculus. The Streptothrix infection was treated by curetting with a small meibomian spoon.

(c) Ectropion of the Lacrimal Punctum.-This condition was seen in ten cases and, unless it is very slight, cauterization behind the punctum or the removal of an ellipse of tissue from that site is necessary.

(d) Functional Epiphora.-In seven patients no objective evidence of epiphora could be seen: one suffered from crocodile tears which began 7 months after a Bell's palsy; two were thought to suffer from paroxysmal hypersecretion due to worry or unusual sensitivity of the conjunctiva; two showed a clear association of anxiety with watering of the eye; and in two a psychogenic disorder was suspected but unconfirmed.

(e) Miscellaneous.-One young boy was seen with tuberculous dacryocystitis. A young man in whom this condition was suspected was found to have a non-specific inflammation when a piece of the sac was removed for histological examination; he had a mucocoele which drained into the throat on pressure over the sac, and a dacryocystorhinostomy was performed with complete success.

A mucocoele of the sac was seen in six women in whom the naso-lacrimal duct could be syringed easily; three were cured by frequent expression, and the restored function of the naso-lacrimal duct was confirmed by the dye test; one patient defaulted; the other two failed to improve. One of the latter also had leukaemia, and it is of interest that two other women with leukaemia and mucocoele of the sac have been seen. It is tempting to conjecture that neoplastic changes in the lymphoid tissue around the nasolacrimal duct may be the cause of the association in these patients.

In one woman aged 35, pain and watering of the eye had been precipitated by emotional upsets; her brother had a watery eye and migraine.

A man aged 50 and a woman aged 61 with endocrine exophthalmos complained of unilateral epiphora. The mechanism of the watering is not known, but appears to rest on a paroxysmal hypersection with impairment of drainage. Syringing of the naso-lacrimal duct could be performed easily in both patients, but fluorescein failed to drain. It may be that the overexposed conjunctivae in these widely-open eyes are subject to an abnormal 
degree of irritation causing lacrimation with a reflex oedema and congestion of the naso-lacrimal pathways. No treatment helped the woman patient and, although a cure of the watering confirmed by the dye test was achieved in the man after a course of exercises, it is likely that this success was due to an incidental spontaneous improvement.

In two men the onset of epiphora followed an attack of herpes zoster ophthalmicus.

In several other cases an obvious reflex cause for watering of the eye was found.

The other cases seen in the course of this investigation have been excluded from discussion, either because of lack of clinical data or because no final assessment of their symptoms has been made.

No patients with congenital stenosis of the duct have been included in this series.

\section{Diagnosis}

The affections described encompass the likely causes of symptomatic epiphora and the following schema may assist in making a diagnosis:

(1) Elimination of gross pathology.

(2) Elimination of obvious causes such as ectropion, ingrowing lashes, etc.

(3) Examination by a rhinologist.

(4) . Consideration of radiological evidence.

(5) Exclusion of affections of the canaliculus by exploratory probing and syringing.

(6) Exclusion of completely blocked naso-lacrimal ducts by a test with pressure syringing.

(7) Investigation of partial blockage of the naso-lacrimal duct by considering the therapeutic benefits of syringing. Patients with a partial blockage, through which it is very difficult to syringe despite occlusion of the upper punctum, do not benefit from pressure syringing.

(8) Consideration of the possibility that the patient has naso-lacrimal catarrh: bilaterality, dye test demonstrating free drainage ( 50 per cent. of cases), naso-lacrimal ducts patent to syringing, slight conjunctival hyperaemia, poor response to syringing, good response to decongestive measures. In male patients naso-lacrimal catarrh is the most common cause of epiphora.

(9) Consideration of the tone of the orbicularis oculi, retraction of the lower lid, and slight eversion of the punctum. A favourable response to a course of orbicularis exercises will tend to confirm the impression that the symptoms are due to "weak muscles".

(10) Demonstration of normal drainage by the dye test and absence of objective evidence of excessive lacrimation indicate that the epiphora may be of functional origin. 
There will still be a number of cases in which the diagnosis will remain in doubt.

\section{Discussion}

The more difficult the diagnosis, the more will some interesting individual detail appear important and obtrusive, so that inclusion in a particular diagnostic group becomes impossible. The undiagnosed cases were grouped with the purpose of avoiding this impasse, in the hope that they would show, when viewed together, some common features. It was expected that patients with weakness of the orbicularis oculi might form such a sub-group, but the existence of the group with "naso-lacrimal catarrh" only became evident as the investigation proceeded.

(1) Sex Differences.-In no group did men preponderate. In patients with completely blocked ducts the sex ratio was more than four females to one male; in patients with partially blocked ducts it was more than two to one, in nasolacrimal catarrh one to one, and in the undiagnosed group three to one. These figures suggest that the condition of "blocked duct" is essentially a disease of women and that the diagnosis of a partial block in some cases was in fact erroneous. The undiagnosed group may also bear some relation to the "blocked ducts", but in only seven was there the slightest difficulty in syringing.

Women tend to lacrimate more than men; they are more subject to emotional weeping and the reddening and congestion of the eyes and conjunctiva, which always accompany a flow of tears, may also affect the naso-lacrimal duct. Emotional upsets without frank lacrimation may also affect the vascularity of the mucous membranes. Women should thus have a tendency to greater variation in the permeability of the lacrimal passages consequent on vascular congestive changes, and this, coupled with a slight but real narrowing of the bony canal as compared with that in men, may reinforce their tendency to be affected by a complete blockage of the naso-lacrimal duct. This leads to the further inference that women should be more subject to naso-lacrimal catarrh, but the figures obtained tend to be weighted in favour of the men who are much more frequently subject to exogenous irritations of the mucous membranes.

The endogenous causes for epiphora are characteristic of women but not exclusively so, and because of the relative narrowing of the bony naso-lacrimal canal, a complete blockage is more frequent in women. On the other hand, exogenous causes are found almost exclusively in men.

(2) Age at Onset.-Although varying slightly in the different groups, the findings indicate that epiphora, whatever its cause, is predominantly an affliction of patients in the 40- to 60-year age group. This applies to both men and women but there also appears to be an additional peak of incidence in men over the age of 65.

Partial blockage of the duct is rarely seen in patients over the age of 54 .

(3) Laterality.-Blocked naso-lacrimal ducts tend to be unilateral and other causes of epiphora bilateral. 
There seems to be little variation in the proportion of unilateral to bilateral cases of blocked ducts (approximately 6:1) between the sexes, but in cases of nasolacrimal catarrh, the men showed a ratio of $1: 3$ and the women $1: 1$, so that in this group, unilaterality is more common in females. In the group of undiagnosed cases the ratio was $1: 1$ for both sexes.

(4) Family History.-A positive family history was obtained in 29 per cent. of cases of completely blocked duct and in 13 per cent. of cases of partially blocked duct, the incidence being similar in both men (6 of 28) and women (17 of 76). A positive family history was also obtained in 11 per cent. of men and in more than 26 per cent. of women with naso-lacrimal catarrh, and in 21 per cent. of women and 10 per cent. of men in whom the epiphora was undiagnosed.

Thus, the occurrence of the symptom in other members of the family is a feature of women with symptomatic epiphora, whatever its cause. In men, a familial incidence of the symptom occurs only when the duct is completely blocked. One may infer from this that a blocked duct is less common in men, because only in certain families do the anatomical predisposing features occur in the male sex. There is a slight tendency for a positive family history to be more common in women with a complete blockage of the naso-lacrimal duct than in the other groups.

(5) Nasal Disorder.-The incidence of nasal disorder is frequent in all groups and may lead to or be associated with an upset in the lacrimal pathway in a proportion of cases. Treatment of the nasal condition is rarely completely successful in alleviating epiphora.

The results of Schirmer's test indicate that a hypersecretion of tears is an additional factor in cases of blocked duct and that this is more marked in patients with a mucocoele. This test is not very reliable and a comparison of the affected side with the unaffected side is probably a better means of interpreting the values obtained. These are shown in Table I, but the evidence is insufficient for any definite conclusions to be drawn.

It is difficult to overestimate the value of the dye test which often indicates the nature of the case within a minute or two. It is also valuable in distinguishing the patient who complains of epiphora despite perfect functioning of the lacrimal apparatus.

Naso-lacrimal catarrh is thought to be the cause of epiphora of many of our patients. The mucous membranes are subject throughout life to the assaults of dust, dirt, smoke, wind, heat, cold, injurious radiations, chemicals, and bacterial contamination from the atmosphere, the fingers, or the nose, but some persons also have an undoubted inherent hypersensitivity of the membranes which may be somatogenic or psychogenic in origin. In this way a psychological upset may provoke an organic defect of tear drainage, and a combination of susceptibility and external sources of injury determines the occurrence of epiphora in many patients.

The basic pathology of blockage of the naso-lacrimal duct has not been determined histologically, but this work, so far as it goes, tends to confirm previous theories that congestion and swelling of the mucous membranes are probably a stage in its causation. 
Conservative treatment often fails to relieve the complaint, and the surgeon undertaking it may often tread as long and as weary a path as the rhinologist attempting to cure nasal catarrh or the ophthalmologist attempting to cure chronic conjunctivitis.

\section{Summary and Conclusions}

Symptomatic epiphora is presented as a clinical and pathological problem.

A suggested scheme of investigation is described. A series of clinical examinations based on such a scheme has revealed two main groups of case other than those of blocked naso-lacrimal duct. One of the group comprised many cases which remained undiagnosed but the other group of cases was postulated as suffering from "naso-lacrimal catarrh", a low-grade congestion and swelling of the mucous membrane of the conjunctiva, canaliculus, lacrimal sac, and/or naso-lacrimal duct.

A clinical analysis of each of these clinical groups led to the following findings:

(1) A few men and a larger number of women are anatomically predisposed to a blockage of the naso-lacrimal duct.

(2) The diagnosis of a blocked naso-lacrimal duct is more common when the condition is unilateral, and is most often seen in females; that of nasolacrimal catarrh is more common when the condition is bilateral, and occurs most frequently in male patients.

(3) Many ducts which are apparently blocked respond to pressure syringing, which is a useful diagnostic and therapeutic procedure and is not considered dangerous.

(4) The conservative treatment of naso-lacrimal catarrh as practised by the author in 36 cases with a demonstrable defect of drainage was followed by objective evidence of cure in sixteen cases.

The author would like to thank the surgeons who have referred cases to his clinic and to express his gratitude to Mr. A. J. B. Goldsmith for his generous help in the preparation of this paper.

\section{REFERENCES}

DUKe-Elder, S. (1952). "'Text-book of Ophthalmology”, vol. 5. Kimpton, London. JACOBS, H. B. (1954). Brit. J. Ophthal., 38, 560. (1959). Ibid., 43, 332.

Phillips, C. I., and George, M. (1956). I Ibid., 40, 673.

SEIDENARI, S. (1947). Minerva Med. (Torino), 38 (1), 181.

SJÖGREN, H. (1943). "A New Conception of Keratoconjunctivitis Sicca". Australian Medical Publishing Co., Sydney.

SondermanN, R. (1923). Klin. Mbl. Augenheilk., 70, 474.

TraQuaIR, H. M. (1940). Trans. ophthal. Soc. U.K., 60, 127.

VALIÈre-VIALEIX, V. (1939). In"Traité d'ophtalmologie", ed. P. Bailliart, C. Coutela, E. Redslob, and E. Velter (publié sous les auspices de la société française d'ophtalmologie), vol. 4, p. 145. Masson, Paris.

Wollenberg, A. (1927). Klin. Mbl. Augenheilk., 78, 825. 